\title{
Effect of Tocilizumab on LDL and HDL Characteristics in Patients with Rheumatoid Arthritis. An Observational Study
}

\author{
Florencia S. Pierini · Eliana Botta · Enrique R. Soriano (D) - Maximiliano Martin • \\ Laura Boero · Tomás Meroño • María Soledad Saez • Ezequiel Lozano Chiappe • \\ Osvaldo Cerda · Gustavo Citera · Ignacio Gandino • Javier Rosa • \\ Patricia Sorroche $\cdot$ Anatol Kontush $\cdot$ Fernando Brites
}

Received: February 2, 2021 / Accepted: March 19, 2021 / Published online: April 2, 2021

(C) The Author(s) 2021

\section{ABSTRACT}

Background: In patients with rheumatoid arthritis (RA), qualitative alterations of low and high-density lipoproteins (LDL and HDL, respectively) might partially explain their increased cardiovascular risk. Tocilizumab has been associated with an increase in lipids, including triglyceride (TG) and cholesterol levels. The aim of this study is to evaluate the effect of tocilizumab on certain LDL and HDL characteristics (oxidized LDL levels, HDL-

F. S. Pierini · E. R. Soriano ( $₫)$ · I. Gandino · J. Rosa Rheumatology Unit, Internal Medical Services, and University Institute, Hospital Italiano de Buenos Aires, Peron 4190, (1181) Buenos Aires, Argentina e-mail: enrique.soriano@hospitalitaliano.org.ar

E. Botta · M. Martin · L. Boero - T. Meroño ·

E. Lozano Chiappe $\cdot$ F. Brites

Laboratory of Lipids and Atherosclerosis, School of Pharmacy and Biochemistry, INFIBIOC, University of Buenos Aires, CONICET, Buenos Aires, Argentina

\section{S. Saez · P. Sorroche}

Central Laboratory, Hospital Italiano de Buenos Aires, Buenos Aires, Argentina

O. Cerda · G. Citera

Rheumatology Service, Instituto de Rehabilitación Psicofísica, Buenos Aires, Argentina

\section{A. Kontush}

Faculty of Medicine Pitié-Salpêtrière, National Institute for Health and Medical Research (INSERM) Research Unit 1166 - ICAN, Sorbonne University, Paris, France associated enzymes, chemical composition of both total HDL and HDL3c subpopulation, and their capacity to promote cellular cholesterol efflux) at baseline and 3 months after the start of treatment in patients with RA.

Methods: Twenty-eight RA patients (ACR/ EULAR 2010 criteria) with indication of treatment with tocilizumab were included in the present study. Clinical assessment [Health assessment questionnaire (HAQ)], disease activity score 28 (DAS28), high-sensitivity C reactive protein (hsCRP) concentration, lipid profile, and lipoprotein (a) [Lp(a)] levels were evaluated in all patients at baseline and after 3 months of treatment with tocilizumab. Lipoprotein characteristics were evaluated through the levels of oxidized LDL (OxLDL), the activity of paraoxonase (PON) 1, the composition of total HDL and small, dense HDL3c subpopulation, and their ability to promote cellular cholesterol efflux.

Results: After 3 months of treatment with tocilizumab, HAQ $(-23 \%, p<0.05)$, DAS28 $(-49 \%, \quad p<0.001)$, and hsCRP $(-94 \%$, $p<0.01)$ levels decreased significantly. Total cholesterol (TC), LDL-C, non-HDL-C, and apo B levels showed a significant increase after treatment (TC: $+7.0 \%, \quad p<0.01 ;$ LDL-C: $+10 \%$, $p<0.01$; non-HDL-C: $+9.9 \%, p<0.01 ;$ and apo B: $+9.6 \%, p<0.05)$. Decreases in $\mathrm{Lp}(\mathrm{a})$ and OxLDL levels were also observed after treatment [Lp(a): $-50 \%, p<0.01$; and oxLDL: $-5.4 \%$, $p<0.05]$. The latter was in accordance with the 
increment detected in PON activity. No changes were observed in HDL capacity to promote cholesterol efflux $(p>0.05)$ in the whole group. Conclusions: Treatment with tocilizumab reduced hsCRP levels and displayed positive effects on certain lipoprotein-related parameters, such as a potent decrease inLp(a) and a reduction in OxLDL levels. Moreover, HDL capacity to promote cellular cholesterol efflux was maintained after 3 months of treatment.

Keywords: Tocilizumab; Rheumatoid arthritis; Lipids; HDL; LDL

\section{Key Summary Points}

Patients with rheumatoid arthritis (RA) presented an increase cardiovascular risk and, although alterations of LDL and HDL lipoproteins only partially explain it. Tocilizumab has been associated with increase in lipids, including triglyceride and cholesterol levels.

The objective of this study was to evaluate the effect of tocilizumab on HDL and LDL characteristics at baseline and 3 months after the start of treatment in patients with RA.

Tocilizumab reduced inflammatory state and displayed positive effects on certain lipoprotein-related parameters, such as a potent decrease in $\operatorname{Lp}(\mathrm{a})$ and a reduction in OxLDL levels and maintained the capacity of total HDL and HDL3c to promote cellular cholesterol efflux.

\section{DIGITAL FEATURES}

This article is published with digital features, including a summary slide, to facilitate understanding of the article. To view digital features for this article go to https://doi.org/10.6084/ m9.figshare.14236802.

\section{INTRODUCTION}

Rheumatoid arthritis (RA) is associated with increased mortality and reduced life expectancy, as a result of accelerated cardiovascular (CV) disease $[1,2]$. Strikingly, CV events occur approximately a decade earlier in RA patients, who are in turn twice more likely to present a myocardial infarction than healthy subjects [3-5]. Traditional CV risk factors, such as dyslipidemia, hypertension, and obesity, do not account for the elevated CV risk in RA [6]. Indeed, classic dyslipidemia, consisting of increased triglyceride and/or low-density lipoprotein (LDL) levels, and/or subnormal concentrations of antiatherogenic high-density lipoproteins (HDL), is scarcely observed in RA patients $[7,8]$. As a corollary, qualitative and functional, rather than quantitative, lipoprotein alterations were proposed to contribute to the increased $\mathrm{CV}$ risk in RA [9-11]. The antiatherogenic functions of HDL primarily involve its capacity to promote cellular cholesterol efflux from peripheral tissues, protection of LDL against oxidative modifications, antiapoptotic effects on endothelial cells, and antiinflammatory actions on the arterial wall [12].

Enhanced cardioprotective effects of HDL are associated with reduced risk of $\mathrm{CV}$ disease, independently of HDL-cholesterol (HDL-C) levels [13]. Small, dense HDL particles possess potent biological activities [40]. We previously evaluated chemical composition and antiatherogenic activity of small, dense HDL3c in active normolipemic RA patients and found that this HDL subpopulation displayed altered lipidome and that its antioxidative activity was reduced according to the degree of inflammation [14]. Chronic inflammation may therefore contribute to increased CV risk in RA via alterations of biological properties of HDL [15].

Tocilizumab is a humanized monoclonal antibody against the interleukin (IL)- 6 receptor(IL-6R), which has shown therapeutic efficacy in RA both as monotherapy and in combination with conventional disease-modifying anti-rheumatic drugs (DMARDs) $[16,17]$. A recent literature search identified eight clinical trials of tocilizumab that reported changes 
in circulating lipids; elevations in serum total cholesterol, HDL-C, LDL-C, and triglyceride levels (if measured) were universally reported [18]. Notably, effects on different atherogenic indexes were inconsistent, but increases in LDLC levels by around 15-20\% were observed in multiple studies, which is consistent with the IL-6-driven lipid changes observed in vivo and in vitro $[19,20]$. The effect of treatment with tocilizumab on qualitative features of LDL and HDL particles in patients with RA has only been superficially studied [21]. Strikingly, in one recent study, increases in triglyceride and LDL$C$ levels by tocilizumab were not associated with an increment in the proportion of atherogenic small, dense LDL and in plasma levels of oxidized LDL [21]. Furthermore, increases in HDL$C$ levels driven by tocilizumab did not imply modifications of its antioxidative properties [22]. Actually, HDL-C levels have been questioned as a therapeutic target for CV risk management, raising the need for more precise and sensitive biomarkers of beneficial effects of HDL [23]. Overall, lipid and lipoprotein plasma concentrations do not reflect the qualitative features of LDL and HDL; this question was not studied in RA patients under therapy with tocilizumab.

Therefore, the aim of this study was to evaluate the effect of tocilizumab on certain LDL and HDL characteristics at baseline and 3 months after the start of treatment in patients with RA. Particularly, oxidized LDL levels, HDLassociated enzymes, chemical composition of both total HDL and HDL3c subpopulation, and their capacity to promote cellular cholesterol efflux were studied.

\section{METHODS}

\section{Study Design and Case Ascertainment}

This was an observational prospective cohort study. Twenty-eight patients with clinical manifestations of RA were recruited at the Rheumatology Unit of Hospital Italiano de Buenos Aires and Instituto de Rehabilitación Psicofísica (IREP) from November 2016 at September 2019. All patients met ACR/EULAR
2010 criteria [24], were over 18 years old, and received tocilizumab de novo administered subcutaneously or intravenously. The indication and prescription were performed by the treating rheumatologist unrelated to this study. The exclusion criteria were diagnosis of diabetes, hypo- or hyper-thyroidism, renal or hepatic alterations, presence of infectious diseases, acute myocardial infarction or stroke during the previous 6 months, and use of antioxidant vitamins or lipid-lowering drugs during the previous months. Special care was taken to avoid including subjects with secondary causes of dyslipidemia such as excessive tobacco smoking (> 20 cigarettes/day) or ethanol consumption ( $>20 \mathrm{~g} /$ day). Illiterate patients or living outside Buenos Aires city area were excluded from the study. Patients were receiving tocilizumab $4 \mathrm{mg} / \mathrm{kg}$ every 4 weeks as an intravenous formulation, which could be increased to $8 \mathrm{mg} / \mathrm{kg}$ according to clinical response [25], or $162 \mathrm{mg}$ tocilizumab subcutaneous injection once per week.

\section{Clinical Assessment}

At the study entry and after 3 months of treatment, disease activity was evaluated by disease activity score using 28 joint counts (DAS28) and functional capacity using the Health assessment questionnaire (HAQ).

\section{Biochemical Parameters}

Biochemical parameters were assessed at baseline and 3 months after treatment with tocilizumab. Plasma levels of total cholesterol (TC), triglycerides (TG), LDL-C, HDL-C, and highsensitivity $C$ reactive protein (hsCRP) were measured using commercially available enzymatic kits. Oxidized LDL (OxLDL) was measured by a commercial ELISA kit (MyBioSource, Inc. San Diego, CA, USA). Apolipoprotein (apo)A-I and apo $B$ concentrations were measured in an IMMAGE nephelometer (Beckman Coulter, Brea, CA, USA). Paraoxonase 1 (PON 1) activity was measured in serum samples employing two different substrates, paraoxon and phenylacetate (Sigma Chemical Co, St. Louis, MO, USA.; 
PON and ARE activities, respectively) following the method of Furlong et al. [26]. Results were expressed as $\mathrm{nmol} / \mathrm{ml} . \mathrm{min}$ and $\mu \mathrm{mol} / \mathrm{ml} . \mathrm{min}$, respectively. Cholesteryl ester transfer protein (CETP) activity was determined in serum samples according to the general procedure previously described by Lagrost et al. [27]. Results were expressed as percentage of ${ }^{3} \mathrm{H}$-cholesteryl ester transferred from HDL3 to apo B-containing lipoproteins, per $\mathrm{ml}$, per hour.

Total HDL (d: $1.063-1.210 \mathrm{~g} / \mathrm{ml})$ and small, dense HDL3c ( $d$ : $1.156-1.179 \mathrm{~g} / \mathrm{ml})$ were isolated from a subset of ten patients by singlestep, isopycnic non-denaturing density gradient ultracentrifugation in a Beckman SW41 Ti rotor at 40,000 rpm for $44 \mathrm{~h}$ in a BeckmanXL70 ultracentrifuge at $15{ }^{\circ} \mathrm{C}$ by a slight modification of the method of Chapman et al. [28] as previously described [29]. Total HDL was prepared by mixing together all HDL subfractions at their equivalent plasma concentrations. Lipoproteins were extensively dialyzed against phosphatebuffered saline (PBS; pH 7.4) at $4{ }^{\circ} \mathrm{C}$ in the dark, stored at $4{ }^{\circ} \mathrm{C}$ and used within 10 days. TC, free cholesterol (FC), TG, and total protein contents of both total HDL and the HDL3c subfraction were determined using commercially available assays. Cholesteryl ester (CE) concentration was calculated by multiplying the difference between total and free cholesterol concentrations by 1.67 [28].

Cholesterol efflux capacity of total HDL and HDL3c was measured in a humanTHP-1 monocytic cell system (obtained from ATCC) at $15 \mu \mathrm{g}$ HDL PL/ml. Assays of cellular cholesterol efflux were performed as previously described with minor modifications [30]. The percentage of cholesterol efflux was calculated as (medium $\mathrm{cpm}) /($ medium $\quad$ cpm + cell cpm $) \times 100 \%$. Specific cholesterol efflux was determined by subtracting non-specific cholesterol efflux occurring in the absence of cholesterol acceptors.

\section{Statistical Analysis}

Categorical variables are reported as frequencies and their 95\% confidence intervals (Cis). Distributions of all variables were analyzed for normality using the Shapiro-Wilk test. Continuous variables are reported as means (SD, standard deviation) or medians (IQR, interquartile range) depending on normally, respectively.

Between-group differences in normally distributed variables were analyzed using paired $t$ test. For non-Gaussian distributed variables, the Wilcoxon matched-pair rank test was used. Differences in dichotomous variables were analyzed by McNemar's test. Correlations between lipoprotein characteristics and acute phase reactants were analyzed by Spearman tests. The statistical software INFOSTAT (National University of Córdoba, Argentina) and SPSS 17.0 (Chicago, IL, USA) were used. A $p$ value of less than 0.05 was considered to be significant.

\section{Sample Size}

Calculation of sample size was done for cholesterol efflux capacity of HDL. To document an increase in this metric of HDL functionality of $25 \%$ after the treatment with tocilizumab with an $80 \%$ power and alpha of 0.05 (two-sided), 25 patients were needed.

\section{Ethical Considerations}

The study was carried out in accordance with Good Clinical Practice (GCP) guidelines, defined in the International Conference on Harmonization (ICH) and in accordance with the ethical principles detailed in the European Union Directive 2001/20/EC and the United States Code of Federal Regulations (Title 21, Part 50 (21CFR50). The study was approved by Institutional Review Board (protocol number 2593).

\section{RESULTS}

Baseline characteristics of the 28 patients included in the study are shown in Table 1 . Most patients were seropositive for rheumatoid factor (RF) and anti-cyclic citrullinated peptide (CCP) antibodies (Table 1). Fourteen patients $(50 \%)$ were receiving tocilizumab as 
Table 1 Baseline characteristics of patients with rheumatoid arthritis $(n=28)$

Age at diagnosis, years, mean (SD)

$44.5(15.2)$

Age at inclusion in the study, years, mean (SD)

$61.3(13.6)$

Female, $n$ (\%, CI 95\%)

$25(89.3,77.1-101.5)$

Disease duration, months, median (IQR)

155 (108-303)

Positive RF, $n$ (\%, CI 95\%)

$19 / 25(76.0,58.0-94.0)$

Positive anti-CCP, $n$ (\%, CI 95\%)

$11 / 17(64.7,39.4-90.0)$

Patients on corticosteroids, $n$ (\%)

$14(50)$

Corticosteroids dose, mg/day, mean (SD)

6.0(4.7)

Body mass index, $n$, mean (SD)

$21,28.0(8.7)$

Concomitant DMARDs, $n(\%)$

$14(50)$

Data are expressed as mean (SD) and as median (IQR), depending on data distribution

$R F$ rheumatoid factor, $C C P$ cyclic citrullinated peptide

monotherapy and 14 patients (50\%) were receiving tocilizumab in combination with DMARDs. Ten patients were receiving methotrexate (MTX), two patients were receiving leflunomide, and two were receiving hydroxychloroquine as DMARD concomitant therapy with tocilizumab. The concomitant consumption of corticoids was taken by 14 (50\%) patients. The corticoid used was methylprednisolone at a mean dose of $6 \mathrm{mg} /$ day (SD 4.7). No medication changes were registered in a 2-week period prior to the blood extraction.

At 3 months of treatment, hsCRP (-94\%, $p<0.01)$, ESR $(-74 \%, p<0.0001)$, DAS28, both calculated with hsCRP $(-47 \%$, $p<0.0001)$ and with ESR $(-50 \%, p<0.0001)$, and HAQ $(-24 \%, p<0.005)$ decreased significantly (Table 2 ).

In parallel, total cholesterol, LDL-C, nonHDL-C, and apo B levels increased significantly after 3 months of treatment (TC: $+7.0 \%$, $p<0.01$; LDL-C: $+10.4 \%, p<0.01$; non-HDL$\mathrm{C}:+9.9 \%, \quad p<0.01 ;$ and apo $\mathrm{B}:+9.6 \%$, $p<0.05)$, while TG levels remained unchanged. In contrast, $\mathrm{Lp}$ (a) decreased $(-50 \%, p<0.001)$ and a reduction in OxLDL concentration was also observed after 3 months of treatment $(-5.4 \%, p<0.05)$. However, no changes were found in HDL-C or in apo A-I levels. Regarding the antioxidant enzyme PON 1, its PON activity was elevated after 3 months of treatment $(p<0.01)$, while its ARE activity did not vary significantly. Moreover, CETP activity also remained unchanged after treatment with tocilizumab (Table 2).

Chemical compositions of total HDL and of its biologically potent subpopulation, HDL3c, were also analyzed. Weight percent chemical composition revealed FC depletion and TG enrichment in total HDL after 3 months of treatment and no significant change in HDL3c (Table 3). Regarding their functionality, neither total HDL nor HDL3c showed significant modifications in the capacity to promote cholesterol efflux from THP-1 cells (Table 4). Nevertheless, changes in this capacity correlated positively with changes in ARE activity $(r=0.69, p<0.05$, respectively) and negatively with hsCRP reduction $(r=-0.70, p<0.05)$.

\section{DISCUSSION}

Consistent with results from randomized control trials with tocilizumab, in the present study, we showed that total cholesterol, LDL-C, non-HDL-C, and apo B levels significantly increased after 3 months of treatment with this drug $[21,31]$. This hypercholesterolemic effect 
Table 2 General characteristics and lipid-related parameters from patients with rheumatoid arthritis $(n=28)$ at baseline and after 3 months of treatment with tocilizumab

\begin{tabular}{llll}
\hline Parameter & Baseline & After 3 months & $p$ for the difference \\
\hline hsCRP $(\mathrm{mg} / \mathrm{l})$ & $3.9(0.6-17.7)$ & $0.5(0.2-1.1)$ & $<0.0001$ \\
ESR $(\mathrm{mm} / \mathrm{h})$ & $33(23-60)$ & $6(2-17)$ & $<0.0001$ \\
DAS 28 (hsCPR) & $4.5(4.1-5.3)$ & $2.1(1.4-3.3)$ & $<0.0001$ \\
DAS 28 (ESR) & $5.4(0.9)$ & $2.8(1.4)$ & $<0.0001$ \\
HAQ & $1.4(1.0-2.0)$ & $1.0(0.9-1.5)$ & $<0.05$ \\
Total cholesterol (mg/dl) & $4.84(0.98)$ & $5.23(1.01)$ & $<0.05$ \\
HDL-C (mg/dl) & $1.48(0.41)$ & $1.53(0.49)$ & $\mathrm{NS}$ \\
LDL-C (mg/dl) & $2.72(0.83)$ & $3.00(0.83)$ & $<0.05$ \\
Non-HDL-C (mg/dl) & $3.37(0.85)$ & $3.70(0.83)$ & $<0.05$ \\
TG $(\mathrm{mg} / \mathrm{dl})$ & $1.33(1.04-1.78)$ & $1.38(1.04-1.98)$ & $\mathrm{NS}$ \\
Apo A-I (mg/dl) & $57.83(47.48-61.76)$ & $55.69(47.12-63.19)$ & $\mathrm{NS}$ \\
Apo B $(\mathrm{mg} / \mathrm{dl})$ & $1.47(0.38)$ & $1.62(0.42)$ & $<0.05$ \\
Lp(a) mg/dl & $48.00(21.60-122.39)$ & $21.60(9.60-64.79)$ & $<0.01$ \\
OxLDL (pg/ml) & $170(147-194)$ & $167(146-175)$ & $<0.05$ \\
PON activity (nmol/ml.min) & $174(70)$ & $191(73)$ & $<0.05$ \\
ARE activity $(\mu \mathrm{mol} / \mathrm{ml} . \mathrm{min})$ & $70(55-86)$ & $71(59-85)$ & $\mathrm{NS}$ \\
CETP activity & $124(29)$ & $108(34)$ & $\mathrm{NS}$ \\
\hline D & & &
\end{tabular}

Data are expressed as mean (SD) and as median (IQR), depending on data distribution

hsCRP high-sensitivity C-reactive protein, ESR erythrocyte sedimentation rate, DAS 28 disease activity score $28, H A Q$ health assessment questionnaire, $H D L-C$ high-density lipoprotein cholesterol, $L D L-C$ low-density lipoprotein cholesterol, $T G$ triglycerides, $O x L D L$ oxidized LDL, $P O N$ paraoxonase, $A R E$ arylesterase, $L p(a)$ lipoprotein (a), CETP cholesteryl ester transfer protein, $N S$ non-significant

caused by tocilizumab has been widely reported and led to the recommendation of monitoring its use particularly in patients with dyslipidemia and high CV risk [32]. However, these modifications could also be interpreted within the context of the "lipid paradox" described for active RA patients [33]. In the chronic inflammatory state of RA, CV risk has been inversely associated with cholesterol levels. This apparently irrational association could be attributed to the fact that treatment with biologic DMARDs are known to effectively reduce the inflammatory state, which in turn leads to a paradoxical elevation of cholesterol levels in response to their anti-inflammatory effect [33]. Moreover, these findings are similar to recent publications from Cacciapaglia et al. [34], Hoffman et al. [35], and Emery et al. [36], who additionally reported an increase in triglycerides levels.

Furthermore, Fioravanti et al. [37] reported only a significant increase in total cholesterol, but beneficial effects on adipokine levels, consisting of a reduction in chemerin and an increase in adiponectin in the subgroups of the patients analyzed. These adipokines are secreted by adipose tissue and present opposite functions. Chemerin was reported to modulate the 
Table 3 Chemical composition of total HDL and small, dense HDL3c subpopulation from patients with rheumatoid arthritis $(n=10)$ at baseline and after 3 months of treatment with tocilizumab

\begin{tabular}{llll}
\hline Component & Group & Total HDL & HDL3c \\
\hline FC (\%) & Baseline & $3.8(0.6)^{*}$ & $2.6(1.6)$ \\
& 3 months & $3.5(0.7)$ & $2.2(1.9)$ \\
CE (\%) & Baseline & $19(3)$ & $12(4)$ \\
& 3 months & $18(3)$ & $11(2)$ \\
TG (\%) & Baseline & $5.6(1.0)^{* *}$ & $5.4(2.1)$ \\
& 3 months & $6.8(1.3)$ & $6.1(1.9)$ \\
PL (\%) & Baseline & $27(23-28)$ & $13(13-16)$ \\
& 3 months & $25(24-27)$ & $15(13-15)$ \\
TP (\%) & Baseline & $46(2)$ & $66(5)$ \\
& 3 months & $48(3)$ & $66(2)$ \\
\hline
\end{tabular}

Data are expressed as mean (SD) and as median (IQR), depending on data distribution. ${ }^{*} p<0.05 ;{ }^{* *} p<0.001$ $H D L$ high-density lipoprotein, $F C$ free cholesterol, $C E$ cholesteryl esters, $T G$ triglycerides, $P L$ phospholipids, $T P$ total proteins

immune system and it is considered responsible for the induction of inflammation and the increase of cardiovascular risk [38]. By contrast, adiponectin is involved in glucose and lipid metabolism with favorable effects on cardiovascular disease [31]. Tocilizumab treatment in RA patients has demonstrated no changes in BMI and fat mass, even though it has been proven to exert positive effects on certain inflammatory and lipoprotein-associated parameters related to adipose tissue. As mentioned by Fioravant et al. [37], these findings might contribute to explain the safety of treatment with tocilizumab related to cardiovascular risk.

On the other hand, the apparently proatherogenic increases in apo B-containing lipoproteins are in contrast to the marked decrease observed in $L p(a)$ plasma levels after 3 months of treatment with tocilizumab. The significant reduction observed in this atherogenic and prothrombotic lipoprotein upon the treatment with tocilizumab is in agreement with findings of different authors [21, 39-41] and can reflect a direct effect of tocilizumab on apo (a) gene expression [42]. Different studies evaluated the impact of biologic DMARDs on Lp(a) levels in patients with RA. Firstly, Schultz et al. [39] reported that the inhibition of the IL6 pathway caused a reduction in $\operatorname{Lp}(\mathrm{a})$ plasma concentrations in a group of patients with rheumatic inflammatory diseases treated with tocilizumab. This finding was already observed after 1 month and it remained up to 3 months of treatment with tocilizumab. Similarly, McInnes et al. [43] found a reduction of $37 \%$ in Lp(a) plasma levels in RA patients treated with tocilizumab plus methotrexate as compared with RA patients treated with placebo plus methotrexate. Moreover, Gabay et al. [44], through a post hoc analysis, showed that Lp(a) plasma levels only decreased in a RA group under treatment with tocilizumab. More recently, a 10-year retrospective study confirmed the above-mentioned findings [41]. Finally, Virone et al. [45] observed the same effect of tocilizumab on $\operatorname{Lp}(\mathrm{a})$ level and other biomarkers, with the exception of a non-significant trend for the deterioration of (increase in) apoB levels. This results were also consistent

Table 4 Cellular cholesterol efflux promoted by total HDL and HDL3c from patients with rheumatoid arthritis $(n=10)$ at baseline and after 3 months of treatment with tocilizumab

\begin{tabular}{llll}
\hline Parameter & Baseline & After 3 months & $\boldsymbol{p}$ for the difference \\
\hline Cholesterol efflux capacity of total HDL (\%) & $4.17(0.99)$ & $3.80(0.73)$ & NS \\
Cholesterol efflux capacity of HDL3c (\%) & $4.38(1.59)$ & $4.81(1.98)$ & NS \\
\hline
\end{tabular}

Data are expressed as mean (SD)

$H D L$ high-density lipoprotein, $N S$ non-significant 
with those obtained by Gabay et al. [44] with a greater improvement of $\mathrm{CV}$ risk-associated biomarkers under tocilizumab than adalimumab treatment. For the first time, after 3 months of treatment with tocilizumab, we were able to detect a reduction in OxLDL, which can be considered as a direct biomarker of the atherosclerotic process [46]. This result could be attributed, at least in part, to the increment detected in PON activity, which significantly contributes to the antioxidative capacity of HDL particles [47]. The study of McInnes et al. [21] also found an increase in PON activity, but failed to detect any variation in OxLDL levels upon IL-6R blockade. CV events are one of the principal causes of death in patients with RA $[1,48,49]$.

Traditional risk factors such as hypertension, smoking, dyslipidemia, and obesity contribute to the endothelial dysfunction in RA, but cannot fully explain the increase in $\mathrm{CV}$ disease. The chronic high-grade inflammatory state of the disease has been linked to the development of premature atherosclerosis. Abnormalities in lipid metabolism, and especially those leading to an increase in LDL levels or to LDL oxidation, play a key role in the atherosclerotic process. Dyslipidemia is common in RA, affecting between 55 and $65 \%$ of patients; however, decreased lipid levels are paradoxically associated with increased $\mathrm{CV}$ risk in $\mathrm{RA}$, whereas levels of LDL-C, HDL-C, and total cholesterol are inversely correlated with markers of chronic inflammation, forming a complex puzzle that results in premature atherosclerosis [50, 51].

Apart from the changes observed in apo B-containing lipoproteins, both beneficial and non-beneficial, no modifications were detected neither in HDL-C, nor in its main apolipoprotein, apo A-I, after treatment with tocilizumab. Recently, "the HDL hypothesis" [52] has been questioned, emphasizing the conclusion that antiatherogenic capacity of HDL is not well reflected by measurement of its cholesterol content [53]. Anti-atherogenic functions of HDL, such as its capacity to promote cellular cholesterol efflux, could better predict future $\mathrm{CV}$ events [54]. Apart from promoting reverse cholesterol transport, whose first step includes cholesterol efflux from peripheral cells, HDL exerts antioxidative properties by inhibiting LDL oxidation as well as anti-inflammatory functions [55]. According to the secondary analysis of the JUPITER (Justification for the Use of Statins in Prevention: an Intervention Evaluating Rosuvastatin) Study, it has also been revealed the relevance of evaluating HDL functionality beyond HDL-C levels. The authors described how HDL functionality, evaluated through HDL inflammatory index, might provide insights into the complex role of HDL, in particular among individuals with chronic inflammation [56].

Among individual HDL subpopulations, small, dense HDL3c is particularly potent in promoting cellular cholesterol efflux and protecting LDL from oxidative damage [57]. Interestingly, atheroprotective properties of HDL are known to be altered in inflammatory diseases including RA and systemic lupus erythematosus $[58,59]$.

When chemical composition of total HDL was evaluated, a reduction in free cholesterol and an increase in triglycerides were detected. The latter was previously associated with an impairment of some cardioprotective functions exerted by HDL in different disorders such as primary hypertriglyceridemia [60], metabolic syndrome [61], and type 2 diabetes [62]. Nevertheless, in this group of RA patients treated with tocilizumab, the above-mentioned compositional alterations of HDL particles did not impact HDL functions. Indeed, HDL capacity to promote cellular cholesterol efflux was unchanged after 3 months of treatment. Two other studies explored cholesterol efflux function of HDL in samples obtained from patients treated with tocilizumab. The one carried out by Ormseth et al. [63] failed to find any difference between baseline and 6 months of treatment in the group of RA patients evaluated and only detected an improvement in the cholesterol efflux capacity after treatment in the subgroup of patients who were initially defective in this functional parameter. On the other hand, Ferraz-Amaro et al. [64] only reported an increase in cholesterol efflux capacity after 6 months of treatment with tocilizumab but not at 3 months. To the best of our knowledge, this is the first time that an HDL subfraction, notably 
small, dense HDL3c, was analyzed in RA patients treated with tocilizumab. Importantly, in HDL3c, which normally possesses potent cardioprotective activities, no adverse alterations were detected. Clinical relevance of small, dense HDL3 was recently highlighted by the studies of Albers et al. [65] and Joshi et al. [66], who observed that CV events showed a stronger association with HDL3 than with total HDL or HDL2 concentrations. Accordingly, the capacity of HDL3c to promote cholesterol efflux was not changed by the treatment with tocilizumab in the present study. No other studies have explored the effect of tocilizumab on small, dense HDL.

Our study has some limitations. The number of patients was limited, and we only assessed the effect at 3 months. However, previous studies [43] have shown little variations on lipids levels after 3 months of therapy with tocilizumab. The low number of patients did not allow us to make subgroup comparisons, however the findings were clear and significant even with this reduced number of patients. We did not have a control group, given that the objective of the study was to compare the change in lipid profile before and after treatment with tocilizumab. We also did not evaluate long-term cardiovascular events, however lipid characteristics are a good surrogate endpoint in cardiovascular disease.

In conclusion, we showed that tocilizumab reduced hsCRP levels and displayed positive effects on certain lipoprotein-related parameters, such as a potent decrease in $\mathrm{Lp}(\mathrm{a})$ levels. Moreover, for the first time, after 3 months of treatment with tocilizumab, a reduction in oxidized LDL concentration was detected, a change that could be attributed to the increment observed in the activity of the HDL-associated antioxidant enzyme PON, thus suggesting that tocilizumab could beneficially modulate certain HDL-atheroprotective functions. Furthermore, no other studies had explored the effect of tocilizumab on chemical composition of both total HDL and small, dense HDL3c particles, and, particularly, the capacity of this active HDL subpopulation to promote cholesterol efflux, which did not suffer any alteration as a consequence of tocilizumab treatment.

So far, when analyzing the balance between pro and antiatherogenic changes, there is no clear evidence of increased CV risk in patients treated with tocilizumab, and the examination of lipoprotein metabolism seems to be consistent with the pattern of decreased risk in spite of elevation of total cholesterol and LDL-C. However, only long-term studies looking at the actual incidence of $\mathrm{CV}$ events would finally resolve this puzzle.

\section{ACKNOWLEDGEMENTS}

The authors thank the patients that agreed to participate for their involvement in the study.

Funding. Study initiated by the researcher supported by Roche Pharmaceuticals. The researcher had full access to all the data and Roche Pharmaceuticals had no role in the study conduction (study design, collection, analysis, and interpretation of the data). No funding or sponsorship was received for the publication of this article.

Authorship. All named authors meet the International Committee of Medical Journal Editors (ICMJE) criteria for authorship for this article, take responsibility for the integrity of the work as a whole, and have given their approval for this version to be published.

Authors' Contributions. All authors made substantial contributions to the intellectual content of this paper. FP, IG, OC, were involved in patient's assessment, and acquisition of data for the work. ERS, JR, GC, and FB were involved in designing the work, analysis, interpretation of data work, and drafting the paper. MM, BL, MT, SMS, LCE and SP were involved in biochemistry tests, and acquisition of data for the work. BE and KA were involved in biochemistry tests, acquisition of data, and drafting the paper. 
Prior Presentation. An abstract of this study was presented at the 2019 ACR/ARP annual meeting on November 8-13, 2019. Atlanta, USA.

Disclosures. PIERINI Florencia S; BOTTA Eliana; MARTIN Maximiliano; BOERO Laura; MEROÑO Tomás; SAEZ María Soledad; LOZANO CHIAPPE Ezequiel; CERDA Osvaldo; SORROCHE Patricia; KONTUSH Anatol and BRITES Fernando declare that they have no conflicts of interest. Enrique R. Soriano: has received grant/research support and/or consulting fees from: AbbVie, Amgen, Bristol-Myers, Squibb, GSK, Genzyme, Janssen, Eli Lilly, Novartis, Pfizer, Roche, Sandoz, Sanofi, UCB. Gustavo Citera: has received grant/research support and/or consulting fees from AbbVie, Amgen, Eli Lilly and Company, GEMA Pharma, Genzyme, Janssen, Novartis, and Pfizer. Javier Rosa: has received consulting fees from AbbVie, Amgen, Eli Lilly, Janssen, Novartis, and Pfizer.

Compliance with Ethics Guidelines. The study was approved by Hospital Italiano Institutional Review Board (protocol number 2593). The study was carried out in accordance with Good Clinical Practice (GCP) guidelines, defined in the International Conference on Harmonization (ICH) and in accordance with the ethical principles detailed in the European Union Directive 2001/20 / EC and the United States Code of Federal Regulations (Title 21, Part 50 (21CFR50), the Helsinki Declaration of 1964 and its later amendments. All patients signed the IRB approved informed consent.

Data Availability. The datasets generated during and/or analyzed during the current study are available from the corresponding author on reasonable request.

Open Access. This article is licensed under a Creative Commons Attribution-NonCommercial 4.0 International License, which permits any non-commercial use, sharing, adaptation, distribution and reproduction in any medium or format, as long as you give appropriate credit to the original author(s) and the source, provide a link to the Creative Commons licence, and indicate if changes were made. The images or other third party material in this article are included in the article's Creative Commons licence, unless indicated otherwise in a credit line to the material. If material is not included in the article's Creative Commons licence and your intended use is not permitted by statutory regulation or exceeds the permitted use, you will need to obtain permission directly from the copyright holder. To view a copy of this licence, visit http://creativecommons.org/licenses/by$\mathrm{nc} / 4.0 /$.

\section{REFERENCES}

1. Gabriel SE. Cardiovascular morbidity and mortality in rheumatoid arthritis. Am J Med. 2008;121(10): S9-14.

2. Di WT, Vergara F, Bertiller E, de LA Gallardo M, Gandino I, Scolnik M, et al. Incidence and prevalence of rheumatoid arthritis in a health management organization in Argentina: a 15-year study. J Rheumatol. 2016;43:1306-11.

3. Jacobsson LTH, Knowler WC, Pillemer S, Hanson RL, Pettitt DJ, Nelson RG, et al. Rheumatoid arthritis and mortality. A longitudinal study in Pima Indians. Arthritis Rheum. 1993;36:1045-53.

4. Semb AG, Kvien TK, Aastveit AH, Jungner I, Pedersen TR, Walldius G, et al. Lipids, myocardial infarction and ischaemic stroke in patients with rheumatoid arthritis in the Apolipoprotein-related Mortality RISk (AMORIS) Study. Ann Rheum Dis. 2010;69:1996-2001.

5. Gabriel SE, Crowson CS, Kremers HM, Doran MF, Turesson C, O'Fallon WM, et al. Survival in rheumatoid arthritis: a population-based analysis of trends over 40 years. Arthritis Rheum. 2003;48: $54-8$.

6. Del Rincón I, Williams K, Stern MP, Freeman GL, Escalante A. High incidence of cardiovascular events in a rheumatoid arthritis cohort not explained by traditional cardiac risk factors. Arthritis Rheum. 2001;44:2737-45 (John Wiley and Sons Inc.).

7. Hahn BH, Grossman J, Ansell BJ, Skaggs BJ, McMahon M. Altered lipoprotein metabolism in chronic inflammatory states: proinflammatory high-density lipoprotein and accelerated atherosclerosis in systemic lupus erythematosus 
and rheumatoid arthritis. Arthritis Res Ther. 2008;10(4):213.

8. Direskeneli H. Excess cardiovascular risk in inflammatory rheumatic diseases: pathophysiology and targeted therapy. Curr Pharm Des. 2012;18:1465-77 (Bentham Science Publishers Ltd.).

9. Tellis CC, Tselepis AD. The role of lipoprotein-associated phospholipase A2 in atherosclerosis may depend on its lipoprotein carrier in plasma. Biochim Biophys Acta. 2009;1791:327-38.

10. Kontush A, Chapman MJ. Functionally defective high-density lipoprotein: a new therapeutic target at the crossroads of dyslipidemia, inflammation, and atherosclerosis. Pharmacol Rev. 2006;58(3): 342-74.

11. Ai M, Otokozawa S, Asztalos BF, Ito Y, Nakajima K, White CC, et al. Small dense LDL cholesterol and coronary heart disease: results from the Framingham offspring study. Clin Chem. 2010;56:967-76.

12. Rye K-A, Barter PJ. Cardioprotective functions of HDLs. J Lipid Res. 2014;55:168-79 (American Society for Biochemistry \& Molecular Biology (ASBMB)).

13. Khera AV, Cuchel M, De La Llera-Moya M, Rodrigues A, Burke MF, Jafri K, et al. Cholesterol efflux capacity, high-density lipoprotein function, and atherosclerosis. N Engl J Med. 2011;364:127-35 (Massachusetts Medical Society).

14. Rosso LG, Lhomme M, Meroño $T$, Sorroche $P$, Catoggio L, Soriano E, et al. Altered lipidome and antioxidative activity of small, dense HDL in normolipidemic rheumatoid arthritis: relevance of inflammation. Atherosclerosis. 2014;237:652-60.

15. Libby P. Inflammation in atherosclerosis. Arterioscler Thromb Vasc Biol. 2012;32:2045-51.

16. Jones G, Sebba A, Gu J, Lowenstein MB, Calvo A, Gomez-Reino JJ, et al. Comparison of tocilizumab monotherapy versus methotrexate monotherapy in patients with moderate to severe rheumatoid arthritis: the AMBITION study. Ann Rheum Dis. 2010;69:88-96.

17. Nishimoto N, Miyasaka N, Yamamoto K, Kawai S, Takeuchi T, Azuma J, et al. Study of active controlled tocilizumab monotherapy for rheumatoid arthritis patients with an inadequate response to methotrexate (SATORI): significant reduction in disease activity and serum vascular endothelial growth factor by IL-6 receptor inhibition t. Mod Rheumatol. 2009;19(1):12-9.

18. Robertson J, Peters MJ, McInnes IB, Sattar N. Changes in lipid levels with inflammation and therapy in RA: a maturing paradigm. Nat Rev Rheumatol. 2013;9(9):513-23.

19. Ettinger WH, Varma VK, Sorci-Thomas M, Parks JS, Sigmon RC, Smith TK, et al. Cytokines decrease apolipoprotein accumulation in medium from Hep G2 cells. Arterioscler Thromb Vasc Biol. 1994;14: 8-13 (Lippincott Williams and Wilkins).

20. Khovidhunkit W, Kim MS, Memon RA, Shigenaga $\mathrm{JK}$, Moser AH, Feingold KR, et al. Effects of infection and inflammation on lipid and lipoprotein metabolism: mechanisms and consequences to the host. J Lipid Res. 2004;45(7):1169-96.

21. McInnes IB, Thompson L, Giles JT, Bathon JM, Salmon JE, Beaulieu AD, et al. Effect of interleukin- 6 receptor blockade on surrogates of vascular risk in rheumatoid arthritis: MEASURE, a randomised, placebo-controlled study. Ann Rheum Dis. 2015;74(4):694-702. https://doi.org/10.1136/annrh eumdis-2013-204345.

22. Strang AC, Bisoendial RJ, Kootte RS, Schulte DM, Dallinga-Thie GM, Levels JHM, et al. Pro-atherogenic lipid changes and decreased hepatic LDL receptor expression by tocilizumab in rheumatoid arthritis. Atherosclerosis. 2013;229:174-81.

23. Toth PP, Barter PJ, Rosenson RS, Boden WE, Chapman MJ, Cuchel M, et al. High-density lipoproteins: a consensus statement from the National Lipid Association. J Clin Lipidol. 2013;7(5):484-525.

24. Aletaha D, Neogi T, Silman AJ, Funovits J, Felson DT, Bingham CO, et al. Rheumatoid arthritis classification criteria: an American College of Rheumatology/European League Against Rheumatism collaborative initiative. Arthritis Rheum. 2010;2010:2569-81 (John Wiley and Sons Inc.).

25. Sheppard M, Laskou F, Stapleton PP, Hadavi S, Dasgupta B. Tocilizumab (Actemra). Hum Vaccines Immunother. 2017;13(9):1972-88 (Taylor and Francis Inc.).

26. Furlong CE, Richter RJ, Seidel SL, Costa LG, Motulsky AG. Spectrophotometric assays for the enzymatic hydrolysis of the active metabolites of chlorpyrifos and parathion by plasma paraoxonase/ arylesterase. Anal Biochem. 1989;180:242-7.

27. Lagrost L, Gandjini H, Athias A, Guyard-Dangremont V, Lallemant C, Gambert P. Influence of plasma cholesteryl ester transfer activity on the LDL and HDL distribution profiles in normolipidemic subjects. Arterioscler Thromb. 1993;13:815-25.

28. Chapman MJ, Goldstein S, Lagrange D, Laplaud PM. A density gradient ultracentrifugal procedure for the isolation of the major lipoprotein classes from human serum. J Lipid Res. 1981;22:339-58. 
29. Guérin M, Bruckert É, Dolphin PJ, Turpin G, Chapman MJ. Fenofibrate reduces plasma cholesteryl ester transfer from HDL to VLDL and normalizes the atherogenic, dense LDL profile in combined hyperlipidemia. Arterioscler Thromb Vasc Biol. 1996;16:763-72 (Lippincott Williams and Wilkins).

30. Villard EF, EI-Khoury P, Frisdal E, Bruckert E, Clement K, Bonnefont-Rousselot D, et al. Genetic determination of plasma cholesterol efflux capacity is gender-specific and independent of HDL-cholesterol levels. Arterioscler Thromb Vasc Biol. 2013;33: 822-8.

31. Toussirot E, Marotte H, Mulleman D, Cormier G, Coury F, Gaudin P, et al. Increased high molecular weight adiponectin and lean mass during tocilizumab treatment in patients with rheumatoid arthritis: a 12-month multicentre study. Arthritis Res Ther. 2020. https://doi.org/10.1186/s13075-02002297-7.

32. Singh JA, Saba B, Lopez-Olivo MA. Tocilizumab for rheumatoid arthritis: a Cochrane systematic review. J Rheumatol. 2011;38:1. https://doi.org/10.3899/ jrheum.100717.

33. Choy E, Sattar N. Interpreting lipid levels in the context of high-grade inflammatory states with a focus on rheumatoid arthritis: a challenge to conventional cardiovascular risk actions. Ann Rheum Dis. 2009;68(4):460-9.

34. Cacciapaglia F, Anelli MG, Rinaldi A, Fornaro M, Lopalco G, Scioscia C, et al. Lipids and atherogenic indices fluctuation in rheumatoid arthritis patients on long-term tocilizumab treatment. Mediators Inflamm. 2018. https://doi.org/10.1155/2018/ 2453265 (Hindawi Limited).

35. Hoffman E, Rahat MA, Feld J, Elias M, Rosner I, Kaly L, et al. Effects of tocilizumab, an anti-interleukin-6 receptor antibody, on serum lipid and adipokine levels in patients with rheumatoid arthritis. Int J Mol Sci. 2019;20(18):4633.

36. Emery P, Rondon J, Parrino J, Lin Y, Pena-Rossi C, Van Hoogstraten $\mathrm{H}$, et al. Safety and tolerability of subcutaneous sarilumab and intravenous tocilizumab in patients with rheumatoid arthritis. Rheumatol (United Kingdom). 2019;58:849-58 (Oxford University Press).

37. Fioravanti A, Tenti S, Bacarelli MR, Damiani A, Li Gobbi F, Bandinelli F, et al. Tocilizumab modulates serum levels of adiponectin and chemerin in patients with rheumatoid arthritis: potential cardiovascular protective role of IL-6 inhibition. Clin Exp Rheumatol. 2019;37:293-300.
38. Makrilakis K, Fragiadaki K, Smith J, Sfikakis PP, Kitas GD. Interrelated reduction of chemerin and plasminogen activator inhibitor-1 serum levels in rheumatoid arthritis after interleukin-6 receptor blockade. Clin Rheumatol. 2015;34:419-27.

39. Schultz O, Oberhauser F, Saech J, Rubbert-Roth A, Hahn M, Krone W, et al. Effects of inhibition of interleukin-6 signalling on insulin sensitivity and lipoprotein (A) levels in human subjects with rheumatoid diseases. PLoS ONE. 2010;5(12): e14328.

40. Gabay C, McInnes IB, Kavanaugh A, Tuckwell K, Klearman M, Pulley J, et al. Comparison of lipid and lipid-associated cardiovascular risk marker changes after treatment with tocilizumab or adalimumab in patients with rheumatoid arthritis. Ann Rheum Dis. 2016;75(10):1806-12.

41. García-Gómez C, Martín-Martínez MA, Castañeda S, Sanchez-Alonso F, Uriarte-Ecenarro M, GonzálezJuanatey C, et al. Lipoprotein(a) concentrations in rheumatoid arthritis on biologic therapy: results from the cardiovascular in rheumatology study project. J Clin Lipidol. 2017;11:749-56 (e3).

42. Müller N, Schulte DM, Türk K, Freitag-Wolf S, Hampe J, Zeuner R, et al. IL-6 blockade by monoclonal antibodies inhibits apolipoprotein (a) expression and lipoprotein (a) synthesis in humans. J Lipid Res. 2015;56:1034-42 (American Society for Biochemistry and Molecular Biology Inc.).

43. McInnes IB, Thompson L, Giles JT, Bathon JM, Salmon JE, Beaulieu AD, et al. Effect of interleukin-6 receptor blockade on surrogates of vascular risk in rheumatoid arthritis: MEASURE, a randomised, placebo-controlled study. Ann Rheum Dis. 2015;74: 694-702 (BMJ Publishing Group).

44. Gabay C, McInnes IB, Kavanaugh A, Tuckwell K, Klearman M, Pulley J, et al. Comparison of lipid and lipid-associated cardiovascular risk marker changes after treatment with tocilizumab or adalimumab in patients with rheumatoid arthritis. Ann Rheum Dis. 2016;75:1806-12.

45. Virone A, Bastard JP, Fellahi S, Capeau J, Rouanet S, Sibilia J, et al. Comparative effect of tumour necrosis factor inhibitors versus other biological agents on cardiovascular risk-associated biomarkers in patients with rheumatoid arthritis. RMD Open. 2019;5(2):e000897.

46. Marchio P, Guerra-Ojeda S, Vila JM, Aldasoro M, Victor VM, Mauricio MD. Targeting early atherosclerosis: a focus on oxidative stress and inflammation. Oxid Med Cell Longev. 2019. https://doi.org/10.1155/2019/8563845 (Hindawi Limited). 
47. Litvinov D, Mahini H, Garelnabi M. Antioxidant and anti-inflammatory role of paraoxonase 1 : implication in arteriosclerosis diseases. $\mathrm{N}$ Am J Med Sci. 2012;4:523-32.

48. Aviña-Zubieta JA, Choi HK, Sadatsafavi M, Etminan M, Esdaile JM, Lacaille D. Risk of cardiovascular mortality in patients with rheumatoid arthritis: a meta-analysis of observational studies. Arthritis Care Res. 2008;59:1690-7.

49. Aviña-Zubieta JA, Thomas J, Sadatsafavi M, Lehman AJ, Lacaille D. Risk of incident cardiovascular events in patients with rheumatoid arthritis: a meta-analysis of observational studies. Ann Rheum Dis. 2012;71:1524-9.

50. Myasoedova E, Crowson CS, Kremers HM, Roger VL, Fitz-Gibbon PD, Therneau TM, et al. Lipid paradox in rheumatoid arthritis: the impact of serum lipid measures and systemic inflammation on the risk of cardiovascular disease. Ann Rheum Dis. 2011;70(3):482-7.

51. Zegkos T, Kitas G, Dimitroulas T. Cardiovascular risk in rheumatoid arthritis: assessment, management and next steps. Ther Adv Musculoskelet Dis. 2016;8:86-101 (SAGE Publications Ltd).

52. Miller GJ, Miller NE. Plasma high-density-lipoprotein concentration and development of ischaemic heart-disease. Lancet. 1975;305:16-9.

53. Camont L, Chapman MJ, Kontush A. Biological activities of HDL subpopulations and their relevance to cardiovascular disease. Trends Mol Med. 2011;17(10):594-603

54. Rohatgi A, Khera A, Berry JD, Givens EG, Ayers CR, Wedin KE, et al. HDL cholesterol efflux capacity and incident cardiovascular events. N Engl J Med. 2014;371:2383-93 (Massachusetts Medical Society).

55. Brites F, Martin M, Guillas I, Kontush A. Antioxidative activity of high-density lipoprotein (HDL): mechanistic insights into potential clinical benefit. BBA Clin. 2017;8:66-77 (Elsevier B.V.).

56. Ajala ON, Demler OV, Liu Y, Farukhi Z, Adelman SJ, Collins HL, et al. Anti-inflammatory HDL function, incident cardiovascular events, and mortality: a secondary analysis of the JUPITER randomized clinical trial. J Am Heart Assoc. 2020;9:e016507.

57. Camont L, Lhomme M, Rached F, Le Goff W, Nègre-Salvayre A, Salvayre R, et al. Small, dense high-density lipoprotein-3 particles are enriched in negatively charged phospholipids: relevance to cellular cholesterol efflux, antioxidative, antithrombotic, anti-inflammatory, and antiapoptotic functionalities. Arterioscler Thromb Vasc Biol. 2013;33(12):2715-23.

58. McMahon M, Grossman J, FitzGerald J, Dahlin-Lee E, Wallace DJ, Thong BY, et al. Proinflammatory high-density lipoprotein as a biomarker for atherosclerosis in patients with systemic lupus erythematosus and rheumatoid arthritis. Arthritis Rheum. 2006;54(8):2541-9.

59. Ronda N, Favari E, Borghi MO, Ingegnoli F, Gerosa $\mathrm{M}$, Chighizola $\mathrm{C}$, et al. Impaired serum cholesterol efflux capacity in rheumatoid arthritis and systemic lupus erythematosus. Ann Rheum Dis. 2014;73: 609-14.

60. Benítez MB, Cuniberti L, Fornari MC, Gómez Rosso L, Berardi V, Elikir G, et al. Endothelial and leukocyte adhesion molecules in primary hypertriglyceridemia. Atherosclerosis. 2008;197:679-87.

61. Gómez Rosso L, Benítez MB, Fornari MC, Berardi V, Lynch S, Schreier L, et al. Alterations in cell adhesion molecules and other biomarkers of cardiovascular disease in patients with metabolic syndrome. Atherosclerosis. 2008;199(2):415-23.

62. Gomez Rosso L, Lhomme M, Meroño T, Dellepiane A, Sorroche P, Hedjazi L, et al. Poor glycemic control in type 2 diabetes enhances functional and compositional alterations of small, dense HDL3c. Biochim Biophys Acta Mol Cell Biol Lipids. 2017;1862:188-95 (Elsevier B.V.).

63. Ormseth MJ, Yancey PG, Solus JF, Louis Bridges S, Curtis JR, Linton MF, et al. Effect of drug therapy on net cholesterol efflux capacity of high-density lipoprotein-enriched serum in rheumatoid arthritis. Arthritis Rheumatol. 2016;68:2099-105 (John Wiley and Sons Inc.).

64. Ferraz-Amaro I, Hernández-Hernández MV, TejeraSegura B, Delgado-Frías E, Macía-Díaz M, Machado JD, et al. Effect of IL-6 receptor blockade on proprotein convertase Subtilisin/Kexin Type-9 and cholesterol efflux capacity in rheumatoid arthritis patients. Horm Metab Res. 2019;51:200-9.

65. Albers JJ, Slee A, Fleg JL, O’Brien KD, Marcovina SM. Relationship of baseline HDL subclasses, small dense LDL and LDL triglyceride to cardiovascular events in the AIM-HIGH clinical trial. Atherosclerosis. 2016;251:454-9 (Elsevier Ireland Ltd).

66. Joshi PH, Toth PP, Lirette ST, Griswold ME, Massaro JM, Martin SS, et al. Association of high-density lipoprotein subclasses and incident coronary heart disease: the Jackson Heart and Framingham Offspring Cohort Studies. Eur J Prev Cardiol. 2016;23: 41-9 (SAGE Publications Inc.). 